\title{
Probing texture zeros with scaling ansatz in inverse seesaw
}

\author{
Ambar Ghosal and Rome Samanta \\ Saha Institute of Nuclear Physics, \\ 1/AF Bidhannagar, Kolkata 700064, India \\ E-mail: ambar.ghosal@saha.ac.in, rome.samanta@saha.ac.in
}

ABSTRACT: We investigate neutrino mass matrix phenomenology involving scaling ansatz and texture zeros adhering inverse seesaw mechanism. It is seen that four is the maximum number of zeros in $m_{D}$ and $\mu$ to obtain viable phenomenology. Depending upon the generic nature of the effective neutrino mass matrices we classify all the emerged matrices in four categories. One of them is ruled out phenomenologically due to inappropriate value of reactor mixing angle after breaking of the scaling ansatz. The mass ordering is inverted in all cases. One of the distinguishable feature of all these categories is the vanishingly small value of $\mathrm{CP}$ violation measure $J_{\mathrm{CP}}$ due to small value of $\delta_{\mathrm{CP}}$. Thus those categories will be ruled out if $\mathrm{CP}$ violation is observed in the leptonic sector in future experiments.

Keywords: Neutrino Physics, CP violation

ARXIV EPRINT: 1501.00916 


\section{Contents}

1 Introduction 1

2 Scaling property and texture zeros 2

34 zero texture 5

4 Parametrization $\quad 7$

5 Phase rotation $\quad 8$

6 Breaking of the scaling ansatz $\quad 9$

$\begin{array}{ll}\text { 6.1 Breaking in the Dirac sector } & 10\end{array}$

$\begin{array}{lll}\text { 6.1.1 Category A,B } & 10\end{array}$

$\begin{array}{lll}\text { 6.1.2 Category C } & 11\end{array}$

$\begin{array}{lll}\text { 6.1.3 Category D } & 12\end{array}$

$\begin{array}{lll}6.2 & \text { Numerical analysis } & 12\end{array}$

$\begin{array}{lll}\text { 6.2.1 Category A,B } & 12\end{array}$

$\begin{array}{lll}6.2 .2 & \text { Category C } & 13\end{array}$

$\begin{array}{lll}6.2 .3 \text { Category D } & 14\end{array}$

$\begin{array}{lll}\text { 6.3 Breaking in Dirac+Majorana sector } & 15\end{array}$

$\begin{array}{lll}\text { 6.3.1 Numerical results } & 15\end{array}$

$\begin{array}{lll}7 & \text { Summary and conclusion } & 17\end{array}$

\section{Introduction}

Among the variants of the seesaw mechanism, inverse seesaw [1-13] stands out as an attractive one, due to its characteristic feature of generation of small neutrino mass without invoking high energy scale in the theory. Although to realize such feature one has to pay the price in terms of incorporation of additional singlet fermions, nevertheless, in different GUT models accommodation of such type of neutral fermions are natural. Furthermore, such mechanism appeals to the foreseeable collider experiments to be testified due to its unique signature. The $9 \times 9$ neutrino mass matrix in this mechanism is written as

$$
m_{\nu}=\left(\begin{array}{ccc}
0 & m_{D} & 0 \\
m_{D}^{T} & 0 & M_{R S} \\
0 & M_{R S}^{T} & \mu
\end{array}\right)
$$


with the choice of basis $\left(\nu_{L}, \nu_{R}^{c}, S_{L}\right)$. The three matrices appear in $m_{\nu}$ are $m_{D}, M_{R S}$ and $\mu$ among them $m_{D}$ and $M_{R S}$ are Dirac type whereas $\mu$ is Majorana type mass matrix. After diagonalization, the low energy effective neutrino mass comes out as

$$
\begin{aligned}
m_{\nu} & =m_{D} M_{R S}^{-1} \mu\left(m_{D} M_{R S}^{-1}\right)^{T} \\
& =F \mu F^{T}
\end{aligned}
$$

where $F=m_{D} M_{R S}^{-1}$. Such definition resembles the above formula as a conventional type-I seesaw expression of $m_{\nu}$. However, $m_{\nu}$ contains large number of parameters and it is possible to fit them with neutrino oscillation experimental data [14-16] (but the predictability is less). Our goal in this work is to find out a phenomenologically viable texture of $m_{D}$ and $\mu$ with minimum number of parameters or equivalently maximum number of zeros. We bring together two theoretical ideas to find out a minimal texture and they are

i) Scaling ansatz [17-27],

ii) Texture Zeros [28-45].

At the outset of the analysis, we choose a basis where the charged lepton mass matrix $\left(m_{E}\right)$ and $M_{R S}$ are diagonal along with texture zeros in $m_{D}$ and $\mu$ matrices. We also start by assuming the scaling property in the elements of $m_{D}$ and $\mu$ to reduce the number of relevant matrices. Although, we are not addressing the explicit origin of such choice of matrices, however, qualitatively we can assume that this can be achieved due to some flavour symmetry [46] which is required to make certain that the texture zeros appear in $m_{D}$ and $\mu$ are in the same basis in which $m_{E}$ and $M_{R S}$ are diagonal. We restrict ourselves within the frame work of $\mathrm{SU}(2)_{L} \times \mathrm{U}(1)_{Y}$ gauge group however, explicit realization of such scheme obviously more elusive which will be studied elsewhere.

\section{Scaling property and texture zeros}

We consider scaling property between the second and third row of $m_{D}$ matrix and the same for $\mu$ matrix also. Explicitly the relationships are written as

$$
\begin{aligned}
\frac{\left(m_{D}\right)_{2 i}}{\left(m_{D}\right)_{3 i}} & =k_{1} \\
\frac{(\mu)_{2 i}}{(\mu)_{3 i}} & =k_{2}
\end{aligned}
$$

where $i=1,2,3$ is the column index. We would like to mention that although we have considered different scale factors for $m_{D}$ and $\mu$ matrices, however, the effective $m_{\nu}$ is still scale invariant and leads to $\theta_{13}=0$. Thus, it is obvious to further break the scaling ansatz. In order to generate nonzero $\theta_{13}$ it is necessary to break the ansatz in $m_{D}$ since, breaking in $\mu$ does not affect the generation of nonzero $\theta_{13}$ although in some cases it provides $m_{3} \neq 0$. In our scheme texture zero format is robust and it remains intact while the scaling ansatz is explicitly broken. Such a scenario can be realized by considering the scaling ansatz and texture zeros to have a different origin. 
Another point is to be noted that, since the $\mu$ matrix is complex symmetric whereas $m_{D}$ is asymmetric, the scale factor considered in $\mu$ matrix is different from that of $m_{D}$ to keep the row wise invariance as dictated by eq. (2.1) (for $m_{D}$ ), and eq. (2.2) (for $\mu$ ). Finally, since the texture of $M_{R S}$ matrix is diagonal it is not possible to accommodate scaling ansatz considered in the present scheme.

Let us now turn to further constrain the matrices assuming zeros in different entries. Since, in our present scheme the matrix $M_{R S}$ is diagonal, we constrain the other two matrices. We start with the maximal zero textures with scaling ansatz of general $3 \times 3$ matrices and list different cases systematically in table 1.

We consider all the matrices ${ }^{1}$ listed in table 1 as the Dirac type matrices $\left(m_{D}\right)$. As the lepton number violating mass matrix $\mu$ is complex symmetric, therefore, the maximal number of zeros with scaling invariance is 5 . Therefore, only $m_{3}^{5}$ and $m_{5}^{5}$ type matrices can be made complex symmetric with the scaling property and are shown in table 2 where they are renamed as $\mu_{1}^{5}$ and $\mu_{2}^{5}$ with a different scale factor $k_{2}$.

Now using eq. (1.2) we can construct $m_{\nu}$ and it is found that all the mass matrices constructed out of these matrices are not suitable to satisfy the neutrino oscillation data. The reason goes as follows:

Case A: $m_{D}(7,6$ zero $)+\mu_{1}^{5}, \mu_{2}^{5}$ (5 zero):

We can not generate nonzero $\theta_{13}$ by breaking the scaling ansatz because in this case all the structures of $m_{D}$ are scaling ansatz invariant. This can be understood in the following way: if we incorporate scaling ansatz breaking by $k_{1}^{\prime} \rightarrow k_{1}(1+\epsilon)$ all the structures of $m_{D}$ are still invariant and $m_{\nu}$ matrix will still give $\theta_{13}=0$ as breaking of scaling in $\mu_{1}^{5}$ and $\mu_{2}^{5}$ play no role for the generation of nonzero value of $\theta_{13}$. To generate nonzero $\theta_{13}$ it is necessary to break scaling ansatz in the Dirac sector.

Case B: $m_{D}(5$ zero $)+\mu_{1}^{5}, \mu_{2}^{5}$ ( 5 zero):

The matrices in the last three rows $\left(m_{4}^{5}\right.$ to $\left.m_{12}^{5}\right)$ of the ' 5 zero texture' part of table 1 are ruled out due to the same reason as mentioned in Case A while, the matrices in the first row i.e. $m_{1}^{5}, m_{2}^{5}$ and $m_{3}^{5}$ give rise to the structure of $m_{\nu}$ as

$$
A_{1}=\left(\begin{array}{ccc}
0 & 0 & 0 \\
0 & * & * \\
0 & * & *
\end{array}\right)
$$

where ' $*$ ' represents some nonzero entries in $m_{\nu}$. This structure leads to complete disappearance of one generation. Moreover it has been shown in ref. [28] that if the number of independent zeros in an effective neutrino mass matrix $\left(m_{\nu}\right)$ is $\geq 3$ it doesn't favour the oscillation data and hence, ' $A_{1}$ ' type mass matrix is ruled out.

Case C: $m_{D}(4$ zero $)+\mu_{1}^{5}$ (5 zero):

There are $12 m_{D}$ matrices with 4 zero texture and they are designated as $m_{1}^{4}, \ldots m_{12}^{4}$ in table 1. Due to the same reason as discussed in Case $\mathbf{A}, m_{10}^{4}, m_{11}^{4}$ and $m_{12}^{4}$ are not

\footnotetext{
${ }^{1}$ From now on we use $m^{n}$ as a mass matrix where $n(=4,5,6,7)$ is the number of zeros in that matrix.
} 


\begin{tabular}{|c|c|c|}
\hline \multicolumn{3}{|c|}{7 zero texture } \\
\hline$m_{1}^{7}=\left(\begin{array}{ccc}0 & 0 & 0 \\
k_{1} c_{1} & 0 & 0 \\
c_{1} & 0 & 0\end{array}\right)$ & $m_{2}^{7}=\left(\begin{array}{ccc}0 & 0 & 0 \\
0 & k_{1} c_{2} & 0 \\
0 & c_{2} & 0\end{array}\right)$ & $m_{3}^{7}=\left(\begin{array}{ccc}0 & 0 & 0 \\
0 & 0 & k_{1} c_{3} \\
0 & 0 & c_{3}\end{array}\right)$ \\
\hline \multicolumn{3}{|c|}{6 zero texture } \\
\hline$m_{1}^{6}=\left(\begin{array}{ccc}d_{1} & 0 & 0 \\
k_{1} c_{1} & 0 & 0 \\
c_{1} & 0 & 0\end{array}\right)$ & $m_{2}^{6}=\left(\begin{array}{ccc}0 & d_{2} & 0 \\
k_{1} c_{1} & 0 & 0 \\
c_{1} & 0 & 0\end{array}\right)$ & $m_{3}^{6}=\left(\begin{array}{ccc}0 & 0 & d_{3} \\
k_{1} c_{1} & 0 & 0 \\
c_{1} & 0 & 0\end{array}\right)$ \\
\hline$m_{4}^{6}=\left(\begin{array}{ccc}d_{1} & 0 & 0 \\
0 & k_{1} c_{2} & 0 \\
0 & c_{2} & 0\end{array}\right)$ & $m_{5}^{6}=\left(\begin{array}{ccc}0 & d_{2} & 0 \\
0 & k_{1} c_{2} & 0 \\
0 & c_{2} & 0\end{array}\right)$ & $m_{6}^{6}=\left(\begin{array}{ccc}0 & 0 & d_{3} \\
0 & k_{1} c_{2} & 0 \\
0 & c_{2} & 0\end{array}\right)$ \\
\hline$m_{7}^{6}=\left(\begin{array}{ccc}d_{1} & 0 & 0 \\
0 & 0 & k_{1} c_{3} \\
0 & 0 & c_{3}\end{array}\right)$ & $m_{8}^{6}=\left(\begin{array}{ccc}0 & d_{2} & 0 \\
0 & 0 & k_{1} c_{3} \\
0 & 0 & c_{3}\end{array}\right)$ & $m_{9}^{6}=\left(\begin{array}{ccc}0 & 0 & d_{3} \\
0 & 0 & k_{1} c_{3} \\
0 & 0 & c_{3}\end{array}\right)$ \\
\hline \multicolumn{3}{|c|}{5 zero texture } \\
\hline$m_{1}^{5}=\left(\begin{array}{ccc}0 & 0 & 0 \\
k_{1} c_{1} & k_{1} c_{2} & 0 \\
c_{1} & c_{2} & 0\end{array}\right)$ & $m_{2}^{5}=\left(\begin{array}{ccc}0 & 0 & 0 \\
k_{1} c_{1} & 0 & k_{1} c_{3} \\
c_{1} & 0 & c_{3}\end{array}\right)$ & $m_{3}^{5}=\left(\begin{array}{ccc}0 & 0 & 0 \\
0 & k_{1} c_{1} & k_{1} c_{3} \\
0 & c_{1} & c_{3}\end{array}\right)$ \\
\hline$m_{4}^{5}=\left(\begin{array}{ccc}d_{1} & d_{2} & 0 \\
k_{1} c_{1} & 0 & 0 \\
c_{1} & 0 & 0\end{array}\right)$ & $m_{5}^{5}=\left(\begin{array}{ccc}0 & d_{2} & d_{3} \\
k_{1} c_{1} & 0 & 0 \\
c_{1} & 0 & 0\end{array}\right)$ & $m_{6}^{5}=\left(\begin{array}{ccc}d_{1} & 0 & d_{3} \\
k_{1} c_{1} & 0 & 0 \\
c_{1} & 0 & 0\end{array}\right)$ \\
\hline$m_{7}^{5}=\left(\begin{array}{ccc}d_{1} & d_{2} & 0 \\
0 & k_{1} c_{2} & 0 \\
0 & c_{2} & 0\end{array}\right)$ & $m_{8}^{5}=\left(\begin{array}{ccc}0 & d_{2} & d_{3} \\
0 & k_{1} c_{2} & 0 \\
0 & c_{2} & 0\end{array}\right)$ & $m_{9}^{5}=\left(\begin{array}{ccc}d_{1} & 0 & d_{3} \\
0 & k_{1} c_{2} & 0 \\
0 & c_{2} & 0\end{array}\right)$ \\
\hline$m_{10}^{5}=\left(\begin{array}{ccc}d_{1} & d_{2} & 0 \\
0 & 0 & k_{1} c_{3} \\
0 & 0 & c_{3}\end{array}\right)$ & $m_{11}^{5}=\left(\begin{array}{ccc}0 & d_{2} & d_{3} \\
0 & 0 & k_{1} c_{3} \\
0 & 0 & c_{3}\end{array}\right)$ & $m_{12}^{5}=\left(\begin{array}{ccc}d_{1} & 0 & d_{3} \\
0 & 0 & k_{1} c_{3} \\
0 & 0 & c_{3}\end{array}\right)$ \\
\hline \multicolumn{3}{|c|}{4 zero texture } \\
\hline$m_{1}^{4}=\left(\begin{array}{ccc}d_{1} & 0 & 0 \\
0 & k_{1} c_{2} & k_{1} c_{3} \\
0 & c_{2} & c_{3}\end{array}\right)$ & $m_{2}^{4}=\left(\begin{array}{ccc}0 & d_{2} & 0 \\
0 & k_{1} c_{2} & k_{1} c_{3} \\
0 & c_{2} & c_{3}\end{array}\right)$ & $m_{3}^{4}=\left(\begin{array}{ccc}0 & 0 & d_{3} \\
0 & k_{1} c_{2} & k_{1} c_{3} \\
0 & c_{2} & c_{3}\end{array}\right)$ \\
\hline$m_{4}^{4}=\left(\begin{array}{ccc}d_{1} & 0 & 0 \\
k_{1} c_{1} & 0 & k_{1} c_{3} \\
c_{1} & 0 & c_{3}\end{array}\right)$ & $m_{5}^{4}=\left(\begin{array}{ccc}0 & d_{2} & 0 \\
k_{1} c_{1} & 0 & k_{1} c_{3} \\
c_{1} & 0 & c_{3}\end{array}\right)$ & $m_{6}^{4}=\left(\begin{array}{ccc}0 & 0 & d_{3} \\
k_{1} c_{1} & 0 & k_{1} c_{3} \\
c_{1} & 0 & c_{3}\end{array}\right)$ \\
\hline$m_{7}^{4}=\left(\begin{array}{ccc}d_{1} & 0 & 0 \\
k_{1} c_{1} & k_{1} c_{2} & 0 \\
c_{1} & c_{2} & 0\end{array}\right)$ & $m_{8}^{4}=\left(\begin{array}{ccc}0 & d_{2} & 0 \\
k_{1} c_{1} & k_{1} c_{2} & 0 \\
c_{1} & c_{2} & 0\end{array}\right)$ & $m_{9}^{4}=\left(\begin{array}{ccc}0 & 0 & d_{3} \\
k_{1} c_{1} & k_{1} c_{2} & 0 \\
c_{1} & c_{2} & 0\end{array}\right)$ \\
\hline$m_{10}^{4}=\left(\begin{array}{ccc}d_{1} & d_{2} & d_{3} \\
k_{1} c_{1} & 0 & 0 \\
c_{1} & 0 & 0\end{array}\right)$ & $m_{11}^{4}=\left(\begin{array}{ccc}d_{1} & d_{2} & d_{3} \\
0 & k_{1} c_{2} & 0 \\
0 & c_{2} & 0\end{array}\right)$ & $m_{12}^{4}=\left(\begin{array}{ccc}d_{1} & d_{2} & d_{3} \\
0 & 0 & k_{1} c_{3} \\
0 & 0 & c_{3}\end{array}\right)$ \\
\hline
\end{tabular}

Table 1. Texture zeros with scaling ansatz of a general $3 \times 3$ matrix. 


$$
\mu_{1}^{5}=\left(\begin{array}{ccc}
0 & 0 & 0 \\
0 & k_{2}^{2} s_{3} & k_{2} s_{3} \\
0 & k_{2} s_{3} & s_{3}
\end{array}\right) \quad \mu_{2}^{5}=\left(\begin{array}{ccc}
0 & k_{2} s_{3} & s_{3} \\
k_{2} s_{3} & 0 & 0 \\
s_{3} & 0 & 0
\end{array}\right)
$$

Table 2. Maximal zero texture of $\mu$ matrix.

considered. Furthermore, $m_{\nu}$ arises through $m_{1}^{4}, m_{4}^{4}$ and $m_{7}^{4}$ also correspond to the ' $A_{1}$ ' type matrix (shown in eq. (2.3)) and hence are also discarded. Finally, remaining six $m_{D}$ matrices $m_{2}^{4}, m_{3}^{4}, m_{5}^{4}, m_{6}^{4}, m_{8}^{4}$ and $m_{9}^{4}$ lead to the structure of $m_{\nu}$ with two zero eigenvalues and obviously they are also neglected.

Case D: $m_{D}(4$ zero $)+\mu_{2}^{5}$ (5 zero):

In this case, for $m_{2}^{4}$ and $m_{3}^{4}$ the low energy mass matrix $m_{\nu}$ comes out as a null matrix while for $m_{1}^{4}$ the structure of $m_{\nu}$ is given by

$$
A_{2}=\left(\begin{array}{ccc}
0 & * & * \\
* & 0 & 0 \\
* & 0 & 0
\end{array}\right)
$$

which is also neglected since the number of independent zeros $\geq 3$. On the other hand rest of the $m_{D}$ matrices $\left(m_{4}^{4}\right.$ to $\left.m_{9}^{4}\right)$ correspond to the structure of $m_{\nu}$ as

$$
A_{3}=\left(\begin{array}{l}
0 * * \\
* * * \\
* * *
\end{array}\right)
$$

Interestingly, a priori we cannot rule out the matrices of type $A_{3}$, however it is observed that $m_{\nu}$ of this type fails to generate $\theta_{13}$ within the present experimental bound (details are mentioned in section 6.2.3). It is also observed that in this scheme to generate viable neutrino oscillation data, four zero texture of both $m_{D}$ and $\mu$ matrices are necessary. Therefore, now on we discuss extensively the four zero texture in both the sectors (Dirac as well as Majorana sector).

\section{4 zero texture}

There are 126 ways to choose 4 zeros out of 9 elements of a general $3 \times 3$ matrix. Hence there are 126 textures. Incorporation of scaling ansatz leads to a drastic reduction to only 12 textures as given in the table 1 . In our chosen basis since $M_{R S}$ is taken as diagonal, therefore, the structure of $m_{D}$ leads to the same structure of $F$. On the other hand the lepton number violating mass matrix $\mu$ is complex symmetric and therefore from the matrices listed in table 1 , only $m_{1}^{4}$ and $m_{10}^{4}$ type matrices are acceptable. We renamed those matrices as $\mu_{1}^{4}$ and $\mu_{2}^{4}$ and explicit structures of them are presented in table 3 . There are now $2 \times 12=24$ types of $m_{\nu}$ due to both the choices of $\mu$ matrices. We discriminate different types of $m_{D}$ matrices in the following way: 


$$
\mu_{1}^{4}=\left(\begin{array}{ccc}
r_{1} & 0 & 0 \\
0 & k_{2}^{2} s_{3} & k_{2} s_{3} \\
0 & k_{2} s_{3} & s_{3}
\end{array}\right) \quad \mu_{2}^{4}=\left(\begin{array}{ccc}
r_{1} & k_{2} s_{3} & s_{3} \\
k_{2} s_{3} & 0 & 0 \\
s_{3} & 0 & 0
\end{array}\right)
$$

Table 3. Four zero texture of $\mu$ matrix.

\begin{tabular}{|c|c|c|c|c|c|c|c|c|c|c|c|c|}
\hline & \multicolumn{10}{|c|}{$m_{D}$} \\
\hline$\mu$ & $m_{1}^{4}$ & $m_{2}^{4}$ & $m_{3}^{4}$ & $m_{4}^{4}$ & $m_{5}^{4}$ & $m_{6}^{4}$ & $m_{7}^{4}$ & $m_{8}^{4}$ & $m_{9}^{4}$ & $m_{10}^{4}$ & $m_{11}^{4}$ & $m_{12}^{4}$ \\
\hline$\mu_{1}^{4}$ & $\times$ & $\times$ & $\times$ & $\checkmark$ & $\checkmark$ & $\checkmark$ & $\checkmark$ & $\checkmark$ & $\checkmark$ & $\times$ & $\times$ & $\times$ \\
\hline$\mu_{2}^{4}$ & $\times$ & $\times$ & $\times$ & $\checkmark$ & $\checkmark$ & $\checkmark$ & $\checkmark$ & $\checkmark$ & $\checkmark$ & $\times$ & $\times$ & $\times$ \\
\hline
\end{tabular}

Table 4. Compositions of the discarded and survived structures of $m_{\nu}$.

\begin{tabular}{|c|c|c|c|c|c|}
\hline$m_{D 1}^{4}=$ & $\left(\begin{array}{ccc}d_{1} & 0 & 0 \\
k_{1} c_{1} & 0 & k_{1} c_{3} \\
c_{1} & 0 & c_{3}\end{array}\right)$ & $m_{D 2}^{4}=$ & $\left(\begin{array}{ccc}0 & d_{2} & 0 \\
k_{1} c_{1} & 0 & k_{1} c_{3} \\
c_{1} & 0 & c_{3}\end{array}\right)$ & $m_{D 3}^{4}=$ & $\left(\begin{array}{ccc}0 & 0 & d_{3} \\
k_{1} c_{1} & 0 & k_{1} c_{3} \\
c_{1} & 0 & c_{3}\end{array}\right)$ \\
\hline$m_{D 4}^{4}=$ & $\left(\begin{array}{ccc}d_{1} & 0 & 0 \\
k_{1} c_{1} & k_{1} c_{2} & 0 \\
c_{1} & c_{2} & 0\end{array}\right)$ & $m_{D 5}^{4}=$ & $\left(\begin{array}{ccc}0 & d_{2} & 0 \\
k_{1} c_{1} & k_{1} c_{2} & 0 \\
c_{1} & c_{2} & 0\end{array}\right)$ & $m_{D 6}^{4}=$ & $\left(\begin{array}{ccc}0 & 0 & d_{3} \\
k_{1} c_{1} & k_{1} c_{2} & 0 \\
c_{1} & c_{2} & 0\end{array}\right)$ \\
\hline
\end{tabular}

Table 5. Four zero textures of the Dirac mass matrices.

i) First of all, the texture $m_{10}^{4}, m_{11}^{4}$ and $m_{12}^{4}$ are always scaling ansatz invariant due to the same reason mentioned earlier in Case $\mathbf{A}$ and hence are all discarded.

Next the matrices $m_{1}^{4}, m_{2}^{4}$ and $m_{3}^{4}$ are also ruled out due to the following:

a) When $\mu_{1}^{4}$ matrix is taken to generate $m_{\nu}$ along with $m_{1}^{4}, m_{2}^{4}$ and $m_{3}^{4}$ as the Dirac matrices, then the structure of the effective $m_{\nu}$ appears such that, one generation is completely decoupled thus leading to two mixing angles zero for the matrix $m_{1}^{4}$ and two zero eigenvalues when we consider $m_{2}^{4}$ and $m_{3}^{4}$ matrices.

b) In case of $\mu_{2}^{4}$ matrix, the form of $m_{\nu}$ for $m_{1}^{4}$ comes out as

$$
A_{4}=\left(\begin{array}{ccc}
* & * & * \\
* & 0 & 0 \\
* & 0 & 0
\end{array}\right)
$$

which is phenomenologically ruled out and for other two matrices $\left(m_{2}^{4}\right.$ and $\left.m_{3}^{4}\right) m_{\nu}$ becomes a null matrix. For a compact view of the above analysis we present the ruled out and survived structures of $m_{\nu}$ symbolically in table 4 .

Thus we are left with same six textures of $m_{D}$ for both the choices of $\mu$ and they are renamed in table 5 as $m_{D 1}^{4}, m_{D 2}^{4}, \ldots m_{D_{6}}^{4}$.

Obviously, it is clear that the above analysis leads to altogether 12 effective $m_{\nu}$ matrices arising due to six $m_{D}\left(m_{D 1}^{4}\right.$ to $\left.m_{D 6}^{4}\right)$ and two $\mu\left(\mu_{1}^{4}\right.$ and $\left.\mu_{2}^{4}\right)$ matrices. 


\begin{tabular}{|c|c|c|c|c|c|c|}
\hline & \multicolumn{2}{|c|}{ Category A } & \multicolumn{4}{|c|}{ Category B } \\
\hline Matrices & $I_{A}$ & $I I_{A}$ & $I_{B}$ & $I I_{B}$ & $I I I_{B}$ & $I V_{B}$ \\
\hline$m_{D}$ & $m_{D 2}^{4}$ & $m_{D 6}^{4}$ & $m_{D 1}^{4}$ & $m_{D 3}^{4}$ & $m_{D 4}^{4}$ & $m_{D 5}^{4}$ \\
\hline$\mu$ & $\mu_{1}^{4}$ & $\mu_{1}^{4}$ & $\mu_{1}^{4}$ & $\mu_{1}^{4}$ & $\mu_{1}^{4}$ & $\mu_{1}^{4}$ \\
\hline
\end{tabular}

Table 6. Different Composition of $m_{D}$ and $\mu_{1}$ matrices to generate $m_{\nu}$.

\begin{tabular}{|c|c|c|c|c|c|c|}
\hline & \multicolumn{2}{|c|}{ Category C } & \multicolumn{4}{|c|}{ Category D } \\
\hline Matrices & $I_{C}$ & $I I_{C}$ & $I_{D}$ & $I I_{D}$ & $I I I_{D}$ & $I V_{D}$ \\
\hline$m_{D}$ & $m_{D 1}^{4}$ & $m_{D 4}^{4}$ & $m_{D 2}^{4}$ & $m_{D 3}^{4}$ & $m_{D 5}^{4}$ & $m_{D 6}^{4}$ \\
\hline$\mu$ & $\mu_{2}^{4}$ & $\mu_{2}^{4}$ & $\mu_{2}^{4}$ & $\mu_{2}^{4}$ & $\mu_{2}^{4}$ & $\mu_{2}^{4}$ \\
\hline
\end{tabular}

Table 7. Different Composition of $m_{D}$ and $\mu_{2}$ matrices to generate $m_{\nu}$.

\section{$4 \quad$ Parametrization}

Depending upon the composition of $m_{D}$ and $\mu$ we subdivided those $12 m_{\nu}$ matrices in four broad categories and each category is again separated in few cases and the decomposition is presented in table 6 and table 7 .

Throughout our analysis we consider the matrix $M_{R S}$ as

$$
M_{R S}=\left(\begin{array}{ccc}
p_{1} & 0 & 0 \\
0 & p_{2} & 0 \\
0 & 0 & p_{3}
\end{array}\right) .
$$

Following eq. (1.2), the $m_{\nu}$ matrix arises in Category A and Category B can be written in a generic way as

$$
m_{\nu}^{A B}=m_{0}\left(\begin{array}{ccc}
1 & k_{1} p & p \\
k_{1} p & k_{1}^{2}\left(q^{2}+p^{2}\right) & k_{1}\left(q^{2}+p^{2}\right) \\
p & k_{1}\left(q^{2}+p^{2}\right) & \left(q^{2}+p^{2}\right)
\end{array}\right)
$$

with the definition of parameters as following

Set $I_{A}: m_{0}^{\prime}=\frac{d_{3}^{2} s_{3}}{p_{3}^{2}}, p^{\prime}=\frac{p_{3} c_{2}}{p_{2} d_{3}}, q^{\prime}=\frac{c_{1} p_{3}}{d_{3} p_{1}} \sqrt{\frac{r_{1}}{s_{3}}}, m_{0}=m_{0}^{\prime}, \quad p=k_{2} p^{\prime}, q=q^{\prime}$

Set $I I_{A}: m_{0}^{\prime}=\frac{d_{2}^{2} s_{3}}{p_{2}^{2}}, p^{\prime}=\frac{p_{2} c_{2}}{p_{3} d_{2}}, q^{\prime}=\frac{c_{1} p_{2}}{d_{2} p_{1}} \sqrt{\frac{r_{1}}{s_{1}}}, m_{0}=m_{0}^{\prime} k_{2}^{2}, p=\frac{p^{\prime}}{k_{2}}, \quad q=\frac{q^{\prime}}{k_{2}}$

Set $I_{B}: m_{0}^{\prime}=\frac{d_{1}^{2} r_{1}}{p_{1}^{2}}, p^{\prime}=\frac{c_{1}}{d_{1}}, \quad q^{\prime}=\frac{c_{3} p_{1}}{d_{1} p_{3}} \sqrt{\frac{s_{3}}{r_{1}}}, m_{0}=m_{0}^{\prime}, \quad p=p^{\prime}, \quad q=q^{\prime}$

Set $I I_{B}: m_{0}^{\prime}=\frac{d_{3}^{2} s_{3}}{p_{3}^{2}}, p^{\prime}=\frac{c_{3}}{d_{3}}, \quad q^{\prime}=\frac{c_{1} p_{3}}{d_{3} p_{1}} \sqrt{\frac{r_{1}}{s_{1}}}, m_{0}=m_{0}^{\prime}, \quad p=p^{\prime}, \quad q=q^{\prime}$

Set $I I I_{B}: m_{0}^{\prime}=\frac{d_{1}^{2} r_{1}}{p_{1}^{2}}, p^{\prime}=\frac{c_{1}}{d_{1}}, \quad q^{\prime}=\frac{c_{2} p_{1}}{d_{1} p_{2}} \sqrt{\frac{s_{3}}{r_{1}}}, m_{0}=m_{0}^{\prime}, \quad p=p^{\prime}, \quad q=k_{2} q^{\prime}$

Set $I V_{B}: m_{0}^{\prime}=\frac{d_{2}^{2} s_{3}}{p_{2}^{2}}, p^{\prime}=\frac{c_{2}}{d_{2}}, \quad q^{\prime}=\frac{c_{1} p_{2}}{d_{2} p_{1}} \sqrt{\frac{r_{1}}{s_{1}}}, m_{0}=m_{0}^{\prime} k_{2}^{2}, p=p^{\prime}, \quad q=\frac{q^{\prime}}{k_{2}}$. 
Similarly the $m_{\nu}$ matrix arises in Category $\mathrm{C}$ can be written as

$$
m_{\nu}^{C}=m_{0}\left(\begin{array}{ccc}
1 & k_{1}(p+q) & p+q \\
k_{1}(p+q) & k_{1}^{2}\left(2 p q+p^{2}\right) & k_{1}\left(2 p q+p^{2}\right) \\
p+q & k_{1}\left(2 p q+p^{2}\right) & \left(2 p q+p^{2}\right)
\end{array}\right)
$$

with the following choice of parameters

$$
\begin{aligned}
& \text { Set } I_{C}: m_{0}^{\prime}=\frac{d_{1}^{2} r_{1}}{p_{1}^{2}}, \quad p^{\prime}=\frac{c_{1}}{d_{1}}, \quad q^{\prime}=\frac{c_{2} p_{1}}{d_{1} p_{2}} \sqrt{\frac{s_{3}}{r_{1}}}, \quad m_{0}=m_{0}^{\prime}, \quad p=p^{\prime}, \quad q=k_{2} q^{\prime} \\
& \text { Set } I I_{C}: m_{0}^{\prime}=\frac{d_{1}^{2} r_{1}}{p_{1}^{2}}, \quad p^{\prime}=\frac{c_{1}}{d_{1}}, \quad q^{\prime}=\frac{c_{3} p_{1}}{d_{1} p_{3}} \sqrt{\frac{s_{3}}{r_{1}}}, \quad m_{0}=m_{0}^{\prime}, \quad p=p^{\prime}, \quad q=q^{\prime} .
\end{aligned}
$$

For Category D the effective $m_{\nu}$ comes out as

$$
m_{\nu}^{D}=m_{0}\left(\begin{array}{ccc}
0 & k_{1} p & p \\
k_{1} p & k_{1}^{2}\left(q^{2}+2 r p\right) & k_{1}\left(q^{2}+2 r p\right) \\
p & k_{1}\left(q^{2}+2 r p\right) & \left(q^{2}+2 r p\right)
\end{array}\right)
$$

with the definition of parameters as

Set $I_{D}: m_{0}^{\prime}=\frac{d_{2}^{2} r_{1}}{p_{1}^{2}}, \quad p^{\prime}=\frac{c_{1} p_{1} s_{3}}{d_{2} p_{2} r_{1}}, q^{\prime}=\frac{c_{1}}{d_{2}}, r^{\prime}=\frac{c_{3}}{d_{2}}, m_{0}=m_{0}^{\prime}, p=k_{2} p^{\prime}, q=q^{\prime}, \quad r=r^{\prime}$

Set $I I_{D}: m_{0}^{\prime}=\frac{d_{3}^{2} r_{1}}{p_{1}^{2}}, \quad p^{\prime}=\frac{c_{1} p_{1} s_{3}}{d_{3} p_{3} r_{1}}, q^{\prime}=\frac{c_{1}}{d_{3}}, r^{\prime}=\frac{c_{2}}{d_{3}}, m_{0}=m_{0}^{\prime}, p=p^{\prime}, \quad q=q^{\prime}, \quad r=k_{2} r^{\prime}$

Set $I I I_{D}: m_{0}^{\prime}=\frac{c_{1} p_{1} s_{3}}{d_{3} p_{3} r_{1}}, p^{\prime}=\frac{c_{1}}{d_{1}}, \quad q^{\prime}=\frac{c_{1}}{d_{3}}, r^{\prime}=\frac{c_{3}}{d_{3}}, m_{0}=m_{0}^{\prime}, p=p^{\prime}, \quad q=k_{2} q^{\prime}, r=r^{\prime}$

Set $I V_{D}: m_{0}^{\prime}=\frac{d_{2}^{2} r_{1}}{p_{1}^{2}}, \quad p^{\prime}=\frac{c_{1} p_{1} s_{3}}{d_{2} p_{2} r_{1}}, q^{\prime}=\frac{c_{1}}{d_{2}}, r^{\prime}=\frac{c_{2}}{d_{2}}, m_{0}=m_{0}^{\prime}, p=k_{2} p^{\prime}, q=q^{\prime}, \quad r=r^{\prime}$

and in general, we consider all the parameters $m_{0}, k_{1}, p, r$ and $q$ are complex.

\section{Phase rotation}

As mentioned earlier, all the parameters of $m_{\nu}$ are complex and therefore we can rephase $m_{\nu}$ by a phase rotation to remove the redundant phases. Here, we systematically study the phase rotation for each category.

Category A,B. The Majorana type mass matrix $m_{\nu}$ can be rotated in phase space through

$$
m_{\nu}^{\prime A B}=P^{T} m_{\nu}^{A B} P
$$

where $P$ is a diagonal phase matrix and is given by $P=\operatorname{diag}\left(e^{i \Phi_{1}}, e^{i \Phi_{2}}, e^{i \Phi_{3}}\right)$.

Redefining the parameters of $m_{\nu}$ as

$$
m_{0} \rightarrow m_{0} e^{i \alpha_{m}}, p \rightarrow p e^{i \theta_{p}}, q \rightarrow q e^{i \theta_{q}}, k_{1} \rightarrow k_{1} e^{i \theta_{1}}
$$


with

$$
\Phi_{1}=-\frac{\alpha_{m}}{2}, \Phi_{2}=-\left(\theta_{1}+\theta_{p}+\frac{\alpha_{m}}{2}\right), \Phi_{3}=-\left(\theta_{p}+\frac{\alpha_{m}}{2}\right)
$$

the phase rotated $m_{\nu}^{\prime A B}$ appears as

$$
m_{\nu}^{\prime A B}=m_{0}\left(\begin{array}{ccc}
1 & k_{1} p & p \\
k_{1} p & k_{1}^{2}\left(q^{2} e^{i \theta}+p^{2}\right) & k_{1}\left(q^{2} e^{i \theta}+p^{2}\right) \\
p & k_{1}\left(q^{2} e^{i \theta}+p^{2}\right) & \left(q^{2} e^{i \theta}+p^{2}\right)
\end{array}\right)
$$

where $\theta=2\left(\theta_{q}-\theta_{p}\right)$ and all the parameters $m_{0}, p, q$ and $k_{1}$ are real. Thus there is only a single phase parameter in $m_{\nu}^{\prime A B}$.

\section{Category C.}

In a similar way, the mass matrix of Category $\mathrm{C}$ can be rephased as

$$
m_{\nu}^{\prime C}=m_{0}\left(\begin{array}{ccc}
1 & k_{1}\left(p+q e^{i \theta}\right) & p+q e^{i \theta} \\
k_{1}\left(p+q e^{i \theta}\right) & k_{1}^{2}\left(2 p q e^{i \theta}+p^{2}\right) & k_{1}\left(2 p q e^{i \theta}+p^{2}\right) \\
p+q e^{i \theta} & k_{1}\left(2 p q e^{i \theta}+p^{2}\right) & \left(2 p q e^{i \theta}+p^{2}\right)
\end{array}\right)
$$

with the same set of redefined parameters as mentioned in eq. (5.2) and (5.3) and the diagonal phase matrix mentioned in the previous case with $\theta=\theta_{q}-\theta_{p}$.

\section{Category D.}

For this category the rephased mass matrix comes out as

$$
m_{\nu}^{\prime D}=m_{0}\left(\begin{array}{ccc}
0 & k_{1} p & p \\
k_{1} p & k_{1}^{2}\left(q^{2} e^{i \alpha}+2 r p e^{i \beta}\right) & k_{1}\left(q^{2} e^{i \alpha}+2 r p e^{i \beta}\right) \\
p & k_{1}\left(q^{2} e^{i \alpha}+2 r p e^{i \beta}\right) & \left(q^{2} e^{i \alpha}+2 r p e^{i \beta}\right)
\end{array}\right)
$$

with $r \rightarrow r e^{i \theta_{r}}, \alpha=2\left(\theta_{q}-\theta_{p}\right), \beta=\left(\theta_{r}-\theta_{p}\right)$ and the rest of the parameters are already defined in eq. (5.2) and eq. (5.3).

\section{Breaking of the scaling ansatz}

Since the neutrino mass matrix obtained in eq. (5.4), (5.5) and (5.6) are all invariant under scaling ansatz and thereby give rise to $\theta_{13}=0$ as well as $m_{3}=0$. Although vanishing value of $m_{3}$ is yet not ruled out however, the former, $\theta_{13}=0$ is refuted by the reactor experimental results. Popular paradigm is to consider $\theta_{13}=0$ at the leading order and by further perturbation nonzero value of $\theta_{13}$ is generated. We follow the same way to produce nonzero $\theta_{13}$ through small breaking of scaling ansatz. It is to be noted in our scheme, generation of nonzero $\theta_{13}$ necessarily needs breaking in $m_{D}$. To generate nonzero $m_{3}$ breaking in $\mu$ matrix is also necessary along with $m_{D}$, however, in Category B since $\operatorname{det}\left(m_{D}=0\right)$ even after breaking in the $\mu$ matrix $m_{\nu}$ still gives one of the eigenvalue equal to zero. On the other hand for Category $\mathrm{C}$ and Category $\mathrm{D}, \mu_{2}^{4}$ has always zero determinant because of being scaling ansatz invariant and therefore, leads to one zero eigenvalue as that of Category B. It is the Category A for which we get nonzero $\theta_{13}$ as well as nonzero $m_{3}$ after breaking the scaling ansatz in both the matrices $\left(m_{D}\right.$ and $\left.\mu\right)$. 
In the following, we invoke breaking of scaling ansatz in all four categories through

i) breaking in the Dirac sector $\left(\theta_{13} \neq 0, m_{3}=0\right)$

ii) breaking in the Dirac sector as well as Majorana sector $\left(\theta_{13} \neq 0, m_{3} \neq 0\right)$ and later we discuss separately both the cases.

\subsection{Breaking in the Dirac sector}

\subsubsection{Category A,B}

We consider minimal breaking of the scaling ansatz through a dimensionless real parameter $\epsilon$ in a single term of different $m_{D}$ matrices of those categories as

$$
m_{D 2}^{4}=\left(\begin{array}{ccc}
0 & d_{2} & 0 \\
k_{1}(1+\epsilon) c_{1} & 0 & k_{1} c_{3} \\
c_{1} & 0 & c_{3}
\end{array}\right), \quad m_{D 6}^{4}=\left(\begin{array}{ccc}
0 & 0 & d_{3} \\
k_{1}(1+\epsilon) c_{1} & k_{1} c_{2} & 0 \\
c_{1} & c_{2} & 0
\end{array}\right)
$$

for Category A and

$$
\begin{aligned}
& m_{D 1}^{4}=\left(\begin{array}{ccc}
d_{1} & 0 & 0 \\
k_{1} c_{1} & 0 & k_{1}(1+\epsilon) c_{3} \\
c_{1} & 0 & c_{3}
\end{array}\right), m_{D 3}^{4}=\left(\begin{array}{ccc}
0 & 0 & d_{3} \\
k_{1}(1+\epsilon) c_{1} & 0 & k_{1} c_{3} \\
c_{1} & 0 & c_{3}
\end{array}\right) \\
& m_{D 4}^{4}=\left(\begin{array}{cccc}
d_{1} & 0 & 0 \\
k_{1} c_{1} & k_{1}(1+\epsilon) c_{2} & 0 \\
c_{1} & c_{2} & 0
\end{array}\right), m_{D 5}^{4}=\left(\begin{array}{ccc}
d_{2} & 0 \\
k_{1}(1+\epsilon) c_{1} & k_{1} c_{2} & 0 \\
c_{1} & c_{2} & 0
\end{array}\right)
\end{aligned}
$$

for Category B. We further want to mention that breaking considered in any element of the second row are all equivalent. For example, if we consider breaking in the '23' element of $m_{D 2}^{4}$ it is equivalent to as considered in eq. (6.1). Neglecting the $\epsilon^{2}$ and higher order terms, the effective $m_{\nu}$ matrix comes out as

$$
m_{\nu}^{\prime A B \epsilon}=m_{0}\left(\begin{array}{ccc}
1 & k_{1} p & p \\
k_{1} p & k_{1}^{2}\left(q^{2} e^{i \theta}+p^{2}\right) & k_{1}\left(q^{2} e^{i \theta}+p^{2}\right) \\
p & k_{1}\left(q^{2} e^{i \theta}+p^{2}\right) & \left(q^{2} e^{i \theta}+p^{2}\right)
\end{array}\right)+m_{0} \epsilon\left(\begin{array}{ccc}
0 & 0 & 0 \\
0 & 2 k_{1}^{2} q^{2} e^{i \theta} & k_{1} q^{2} e^{i \theta} \\
0 & k_{1} q^{2} e^{i \theta} & 0
\end{array}\right)
$$

As mentioned earlier, that for Category $\mathrm{B}, \operatorname{det}\left(m_{D}\right)=0$ and it is not possible to generate $m_{3} \neq 0$ even if we consider breaking in the $\mu$ matrices. On the other hand, the matrices in Category A posses $\operatorname{det}\left(m_{D}\right) \neq 0$ and thereby give rise to $m_{3} \neq 0$.

Now to calculate the eigenvalues, mixing angles, $J_{\mathrm{CP}}$, the Dirac and Majorana phases we utilize the results obtained in ref. [47], for a general complex matrix. We should mention that the formula obtained in ref. [47], for Majorana phases is valid when all three eigenvalues are nonzero. However, when one of the eigenvalue is zero (in this case $m_{3}=0$ ) one has to utilize the methodology given in ref. [18], which shows, a general Majorana type mass matrix $m_{\nu}$ can be diagonalized as

$$
U^{\dagger} m_{\nu} U^{*}=\operatorname{diag}\left(m_{1}, m_{2}, m_{3}\right)
$$


or alternely,

$$
m_{\nu}=\operatorname{Udiag}\left(m_{1}, m_{2}, m_{3}\right) U^{T}
$$

where

$$
U=U_{C K M} P_{M}
$$

The mixing matrix $U_{C K M}$ is given by (following PDG [48]) convention)

$$
U_{C K M}=\left(\begin{array}{ccc}
c_{12} c_{13} & s_{12} c_{13} & s_{13} e^{-i \delta_{c p}} \\
-s_{12} c_{23}-c_{12} s_{23} s_{13} e^{i \delta_{c p}} & c_{12} c_{23}-s_{12} s_{13} s_{23} e^{i \delta_{c p}} & c_{13} s_{23} \\
s_{12} s_{23}-c_{12} s_{13} c_{23} e^{i \delta_{c p}} & -c_{12} s_{23}-s_{12} s_{13} c_{23} e^{i \delta_{c p}} & c_{13} c_{23}
\end{array}\right)
$$

with $c_{i j}=\cos \theta_{i j}, s_{i j}=\sin \theta_{i j}$ and $\delta_{\mathrm{CP}}$ is the Dirac CP phase. The diagonal phase matrix $P_{M}$ is parametrized as

$$
P_{M}=\operatorname{diag}\left(1, e^{\alpha_{M}}, e^{i\left(\beta_{M}+\delta_{\mathrm{CP}}\right)}\right)
$$

with $\alpha_{M}$ and $\beta_{M}+\delta_{\mathrm{CP}}$ are the Majorana phases.

Writing eq. (6.5) explicitly with $m_{3}=0$ we can have expressions for six independent elements of $m_{\nu}$ in terms of the mixing angles, two eigenvalues and the Dirac CP phase, from which the $m_{11}$ element can be expressed as

$$
m_{11}=c_{12}^{2} c_{13}^{2} m_{1}+s_{12}^{2} c_{13}^{2} m_{2} e^{2 i \alpha_{M}}
$$

and therefore the Majorana phase $\alpha_{M}$ comes out as

$$
\alpha_{M}=\frac{1}{2} \cos ^{-1}\left\{\frac{\left|m_{11}\right|^{2}}{2 c_{12}^{2} s_{12}^{2} c_{13}^{4} m_{1} m_{2}}-\frac{\left(c_{12}^{4} m_{1}^{2}+s_{12}^{4} m_{2}^{2}\right)}{2 c_{12}^{2} s_{12}^{2} m_{1} m_{2}}\right\} .
$$

The Jarlskog measure of CP violation $J_{\mathrm{CP}}$ is defined in usual way as

$$
J_{\mathrm{CP}}=\frac{I m\left(h_{12} h_{23} h_{31}\right)}{\left(\Delta m_{21}^{2}\right)\left(\Delta m_{32}^{2}\right)\left(\Delta m_{31}^{2}\right)}
$$

where $h$ is a hermitian matrix constructed out of $m_{\nu}$ as $h=m_{\nu} m_{\nu}^{\dagger}$.

\subsubsection{Category $\mathrm{C}$}

In this case breaking is considered in $m_{D}$ as

$$
m_{D 1}^{4}=\left(\begin{array}{ccc}
d_{1} & 0 & 0 \\
k_{1}(1+\epsilon) c_{1} & k_{1} c_{2} & 0 \\
c_{1} & c_{2} & 0
\end{array}\right), \quad m_{D 4}^{4}=\left(\begin{array}{ccc}
d_{1} & 0 & 0 \\
k_{1}(1+\epsilon) c_{1} & 0 & k_{1} c_{3} \\
c_{1} & 0 & c_{3}
\end{array}\right)
$$

and the scaling ansatz broken $m_{\nu}$ appears as

$$
\begin{aligned}
m_{\nu}^{\prime C \epsilon}= & m_{0}\left(\begin{array}{ccc}
1 & k_{1}\left(p+q e^{i \theta}\right) & p+q e^{i \theta} \\
k_{1}\left(p+q e^{i \theta}\right) & k_{1}^{2}\left(2 p q e^{i \theta}+p^{2}\right) & k_{1}\left(2 p q e^{i \theta}+p^{2}\right) \\
p+q e^{i \theta} & k_{1}\left(2 p q e^{i \theta}+p^{2}\right) & \left(2 p q e^{i \theta}+p^{2}\right)
\end{array}\right) \\
& +m_{0} \epsilon\left(\begin{array}{ccc}
0 & k_{1} q e^{i \theta} & 0 \\
k_{1} q e^{i \theta} & 2 k_{1}^{2} p q e^{i \theta} & k_{1} p q e^{i \theta} \\
0 & k_{1} p q e^{i \theta} & 0
\end{array}\right) .
\end{aligned}
$$




\begin{tabular}{|c|c|}
\hline Quantity & $3 \sigma$ ranges \\
\hline$\left|\Delta m_{31}^{2}\right| \mathrm{N}$ & $2.31<\Delta m_{31}^{2}\left(10^{3} \mathrm{eV}^{-2}\right)<2.74$ \\
\hline$\left|\Delta m_{31}^{2}\right| \mathrm{I}$ & $2.21<\Delta m_{31}^{2}\left(10^{3} \mathrm{eV}^{-2}\right)<2.64$ \\
\hline$\Delta m_{21}^{2}$ & $7.21<\Delta m_{21}^{2}\left(10^{5} \mathrm{eV}^{-2}\right)<8.20$ \\
\hline$\theta_{12}$ & $31.3^{\circ}<\theta_{12}<37.46^{\circ}$ \\
\hline$\theta_{23}$ & $36.86^{\circ}<\theta_{23}<55.55^{\circ}$ \\
\hline$\theta_{13}$ & $7.49^{\circ}<\theta_{13}<10.46^{\circ}$ \\
\hline
\end{tabular}

Table 8. Input experimental values [16].

\subsubsection{Category D}

Breaking in $m_{D}$ in this case is incorporated through

$$
\begin{array}{rlrl}
m_{D 2}^{4}= & \left(\begin{array}{ccc}
0 & d_{2} & 0 \\
k_{1} c_{1} & 0 & k_{1}(1+\epsilon) c_{3} \\
c_{1} & 0 & c_{3}
\end{array}\right), & m_{D 3}^{4}=\left(\begin{array}{ccc}
0 & 0 & d_{3} \\
k_{1} c_{1} & 0 & k_{1}(1+\epsilon) c_{3} \\
c_{1} & 0 & c_{3}
\end{array}\right) \\
m_{D 5}^{4}=\left(\begin{array}{cccc}
0 & d_{2} & 0 \\
k_{1} c_{1} & k_{1}(1+\epsilon) c_{2} & 0 \\
c_{1} & c_{2} & 0
\end{array}\right), & m_{D 6}^{4}=\left(\begin{array}{ccc}
0 & 0 & d_{3} \\
k_{1} c_{1} & k_{1}(1+\epsilon) c_{2} & 0 \\
c_{1} & c_{2} & 0
\end{array}\right)
\end{array}
$$

and the corresponding $m_{\nu}$ comes out as

$$
\begin{aligned}
m_{\nu}^{\prime D \epsilon}= & m_{0}\left(\begin{array}{ccc}
0 & k_{1} p & p \\
k_{1} p & k_{1}^{2}\left(q^{2} e^{i \alpha}+2 r p e^{i \beta}\right) & k_{1}\left(q^{2} e^{i \alpha}+2 r p e^{i \beta}\right) \\
p & k_{1}\left(q^{2} e^{i \alpha}+2 r p e^{i \beta}\right) & \left(q^{2} e^{i \alpha}+2 r p e^{i \beta}\right)
\end{array}\right) \\
& +m_{0} \epsilon\left(\begin{array}{ccc}
0 & 0 & 0 \\
0 & 2 k_{1}^{2} r p e^{i \beta} & k_{1} r p e^{i \beta} \\
0 & k_{1} r p e^{i \beta} & 0
\end{array}\right) .
\end{aligned}
$$

\subsection{Numerical analysis}

In order to perform the numerical analysis to obtain allowed parameter space we utilize the neutrino oscillation data obtained from global fit shown in table 8 .

\subsubsection{Category A,B}

We first consider Category A,B for which the neutrino mass matrix is given in eq. (6.3). The parameter $\epsilon$ is varied freely to fit the extant data and it is constrained as $0.04<\epsilon<0.7$. However, to keep the ansatz breaking effect small we restrict the value of $\epsilon$ only upto 0.1 . For this range of $\epsilon(0<\epsilon<0.1)$ under consideration the parameter spaces are obtained as $1.78<p<3.40,1.76<q<3.42$ and $0.66<k_{1}<1.3$. It is interesting to note a typical feature of this category is that the Dirac CP phase $\delta_{\mathrm{CP}}$ comes out too tiny and thereby generating almost vanishing value of $J_{\mathrm{CP}}\left(\approx 10^{-6}\right)$ while the range of the only Majorana phase in this category is obtained as $77^{\circ}<\alpha_{M}<90^{\circ}$.

As one of the eigenvalue $m_{3}=0$ therefore, the hierarchy of the masses is clearly inverted in this category. The sum of the three neutrino masses $\Sigma_{i} m_{i}\left(=m_{1}+m_{2}+m_{3}\right)$ 

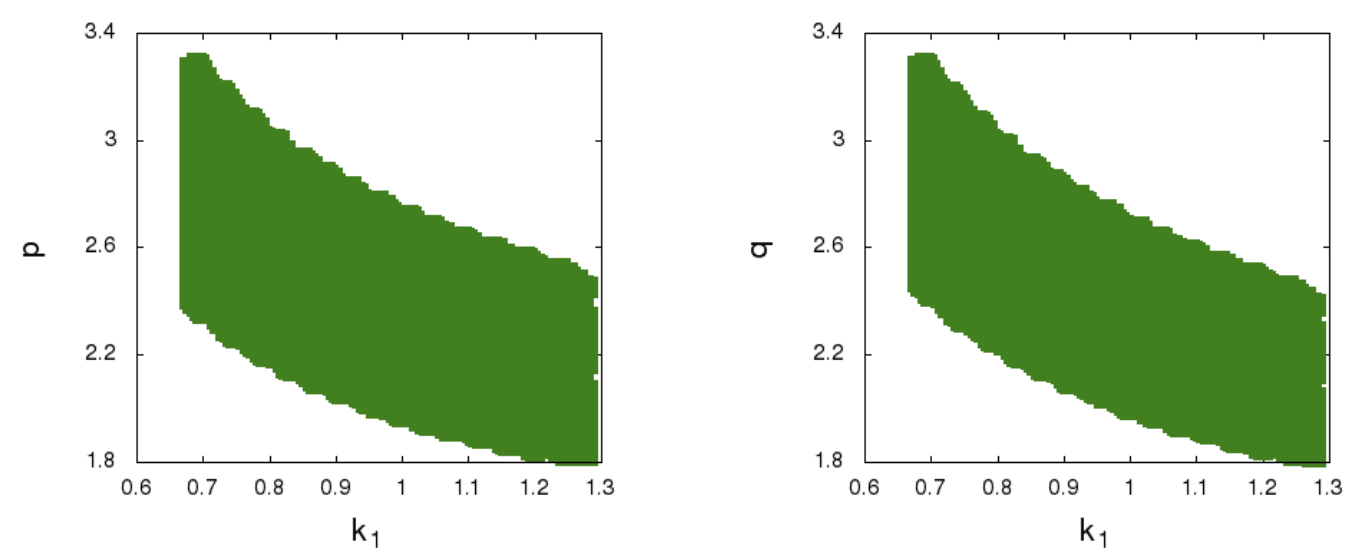

Figure 1. Plot of $p$ vs $k_{1}$ (left), $q$ vs $k_{1}$ (right) for the Category A,B with $\epsilon=0.1$..

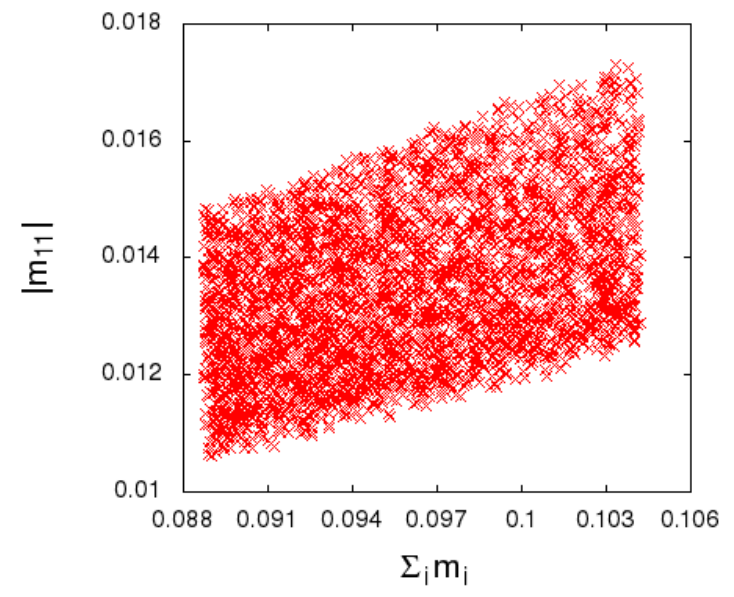

Figure 2. Plot of $\left|m_{11}\right|$ vs $\Sigma_{i} m_{i}$ for Category A,B with $\epsilon=0.1$.

and $\left|m_{11}\right|$ are obtained as $0.088 \mathrm{eV}<\Sigma_{i} m_{i}<0.104 \mathrm{eV}$ and $0.0102 \mathrm{eV}<\left|m_{11}\right|<0.0181 \mathrm{eV}$ which predict the value of the two quantities below the present experimental upper bounds. To illustrate the nature of variation, in figure 1 we plot $p$ vs $k_{1}$ and $q$ vs $k_{1}$ while in figure 2 a correlation plot of $\Sigma_{i} m_{i}$ with $\left|m_{11}\right|$ is shown for $\epsilon=0.1$ and it is also seen from figure 1 and 2 that the ranges of the parameters do not differ much compare to the values obtained for the whole range of $\epsilon$ parameter.

In brief, distinguishable characteristics of this category are i) tiny $J_{\mathrm{CP}}$ and $\delta_{\mathrm{CP}}$ ii) inverted hierarchy of the neutrino masses. At the end of this section we will further discuss the experimental testability of these quantities for all the categories.

\subsubsection{Category $\mathrm{C}$}

In this case it is found that a small breaking of $\epsilon(0.02<\epsilon<0.09)$ is sufficient to accommodate all the oscillation data. We explore the parameter space and the ranges obtained as $3.42<p<6.07,1.68<q<3.02$ and $0.7<k_{1}<1.32$. The hierarchy obtained in this 

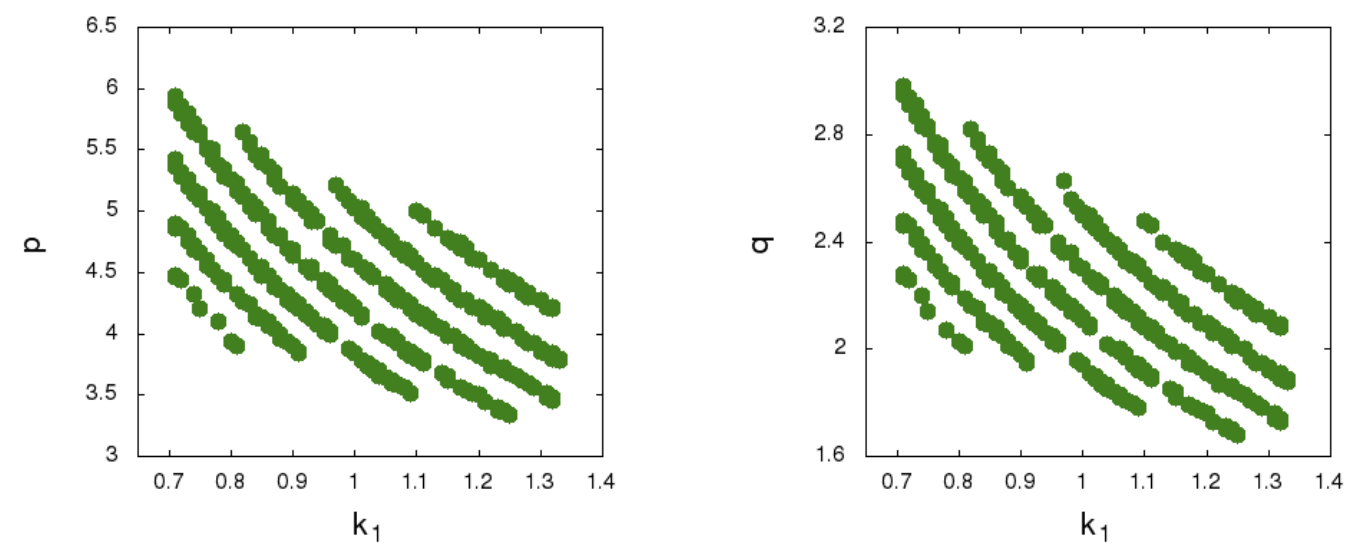

Figure 3. Plot of $p$ vs $k_{1}$ (left), $q$ vs $k_{1}$ (right) for the Category $\mathrm{C}$ with $\epsilon=0.09$.

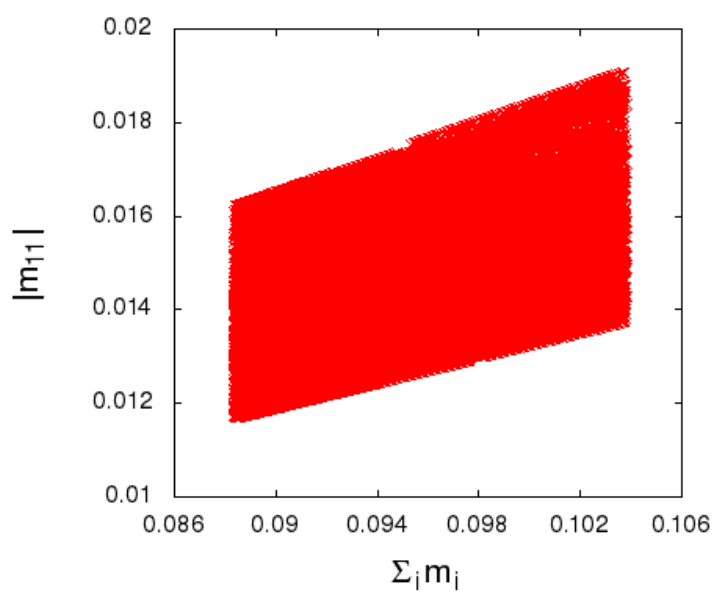

Figure 4. Plot of $\left|m_{11}\right|$ vs $\Sigma_{i} m_{i}$ for Category C with $\epsilon=0.09$.

case is also inverted due to the vanishing value of $m_{3}$. The other two quantities $\Sigma_{i} m_{i}$ and $\left|m_{11}\right|$ come out as $0.0118 \mathrm{eV}<\left|m_{11}\right|<0.019 \mathrm{eV}$ and $0.088 \mathrm{eV}<\Sigma_{i} m_{i}<0.105 \mathrm{eV}$. Similar to the previous category $J_{\mathrm{CP}}$ is vanishingly small due to low value of $\delta_{\mathrm{CP}}$. The range of the Majorana phase $\alpha_{M}$ is obtained as $81^{\circ}<\alpha_{M}<89^{\circ}$. In figure 3 we plot $k_{1}$ vs $p$ and $k_{1}$ vs $q$ for $\epsilon=0.09$ that predicts almost the same ranges of the parameters $\left(p, q\right.$ and $\left.k_{1}\right)$ and all other quantities $\left(\left|m_{11}\right|, \Sigma_{i} m_{i}, \alpha_{M}\right.$ and $\left.J_{\mathrm{CP}}\right)$ as obtained from the whole range of $\epsilon$. We present a correlation plot of $\Sigma_{i} m_{i}$ with $\left|m_{11}\right|$ in figure 4.

\subsubsection{Category D}

In case of Category $\mathrm{D}$, although a priori it is not possible to rule out $m_{\nu}^{\prime D \epsilon}$ without going into the detailed numerical analysis, however in this case even if with $\epsilon=1$ it is not possible to accommodate the neutrino oscillation data. Specifically, the value of $\theta_{13}$ is always beyond the reach of the parameter space. Exactly for the same reason the $m_{\nu}$ matrix of type $A_{3}$ in eq. (2.5) is phenomenologically ruled out. 


\subsection{Breaking in Dirac+Majorana sector}

In this section we focus on the phenomenology of the neutrino mass matrix where the scaling ansatz is broken in both the sectors. This type of breaking is only relevant for Category $\mathrm{A}$ since in this case $m_{D}$ is nonsingular after breaking of the ansatz and the resultant $m_{\nu}$ gives rise to nonzero $\theta_{13}$ along with $m_{3} \neq 0$. In all the other categories due to the singular nature of $m_{D}$, inclusion of symmetry breaking in the Majorana sector will not generate $m_{3} \neq 0$. Thus we consider only Category A under this scheme.

We consider the breaking in $m_{D}$ as mentioned in eq. (6.1) and the ansatz broken texture of $\mu_{1}^{4}$ matrix is given by

$$
\mu_{1}^{4}=\left(\begin{array}{ccc}
r_{1} & 0 & 0 \\
0 & k_{2}^{2} s_{3} & k_{2}\left(1+\epsilon^{\prime}\right) s_{3} \\
0 & k_{2}\left(1+\epsilon^{\prime}\right) s_{3} & s_{3}
\end{array}\right)
$$

where $\epsilon^{\prime}$ is a dimensionless real parameter. The effective neutrino mass matrix $m_{\nu}$ comes out as

$$
\begin{aligned}
m_{\nu \epsilon^{\prime}}^{\prime A \epsilon}= & m_{0}\left(\begin{array}{ccc}
1 & k_{1} p & p \\
k_{1} p & k_{1}^{2}\left(q^{2} e^{i \theta}+p^{2}\right) & k_{1}\left(q^{2} e^{i \theta}+p^{2}\right) \\
p & k_{1}\left(q^{2} e^{i \theta}+p^{2}\right) & \left(q^{2} e^{i \theta}+p^{2}\right)
\end{array}\right)+m_{0} \epsilon\left(\begin{array}{ccc}
0 & 0 & 0 \\
0 & 2 k_{1}^{2} q^{2} e^{i \theta} & k_{1} q^{2} e^{i \theta} \\
0 & k_{1} q^{2} e^{i \theta} & 0
\end{array}\right) \\
& +m_{0} \epsilon^{\prime}\left(\begin{array}{cccc}
0 & k_{1} p & p \\
k_{1} p & 0 & 0 \\
p & 0 & 0
\end{array}\right) .
\end{aligned}
$$

\subsubsection{Numerical results}

As mentioned above, $\epsilon^{\prime}=0$ leads to inverted hierarchy with $m_{3}=0$ and thus to generate nonzero $m_{3}$ a small value of $\epsilon^{\prime}$ is needed. Similar to the previous cases two breaking parameters $\epsilon$ and $\epsilon^{\prime}$ can be varied freely through the ranges that are sensitive to the oscillation data and are obtained as $0.06<\epsilon<0.68$ and $0<\epsilon^{\prime}<1$. It is to be noted that although the $\epsilon$ parameter is restricted due to $\theta_{13}$ value, $\epsilon^{\prime}$ is almost insensitive to $\theta_{13}$ and it can vary within a wide range as $0<\epsilon^{\prime}<1$. A correlation plot of $\epsilon$ with $\epsilon^{\prime}$ is shown in figure 5. However, as mentioned earlier, the effect of the breaking term should be smaller than the unbroken one, therefore, to obtain the parameter space for this category we consider breaking of the scaling ansatz in both the sectors only upto $10 \%$ and consequently for all combinatorial values of $\epsilon$ and $\epsilon^{\prime}$ the parameters $p, q$ and $k_{1}$ vary within the ranges as $1.07<p<3.10,1.03<q<3.12$ and $0.67<k_{1}<1.31$. Interestingly, although all the eigenvalues are nonzero in this case, the hierarchy is still inverted. $J_{\mathrm{CP}}$ is found to be tiny $\left(\approx 10^{-6}\right)$ again due to small value of $\delta_{\mathrm{CP}}$. The Majorana phases are obtained as $-96^{\circ}<\alpha_{M}<74^{\circ}$ and $-100^{\circ}<\beta_{M}+\delta_{\mathrm{CP}}<102^{\circ}$ followed by the bounds on $\Sigma_{i} m_{i}$ and $\left|m_{11}\right|$ as $0.088 \mathrm{eV}<\Sigma_{i} m_{i}<0.11 \mathrm{eV}$ and $0.010 \mathrm{eV}<\left|m_{11}\right|<0.022 \mathrm{eV}$ which are well below the present experimental upper bounds. In figure 6 we demonstrate the above predictions for $\epsilon=\epsilon^{\prime}=0.1$. In the left panel of figure 6 the inverted hierarchical nature is shown and in the right panel variation of the Majorana phases is demonstrated. Some comments are in order regarding predictions of the present scheme: 


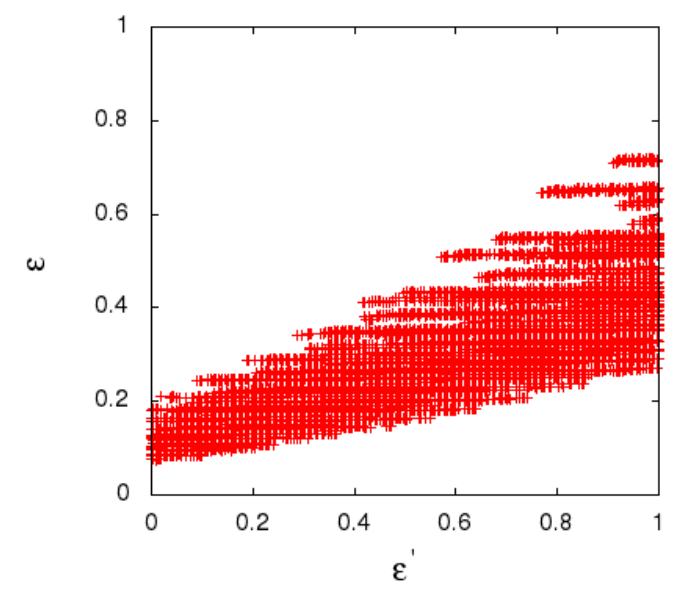

Figure 5. Correlated plot of $\epsilon$ with $\epsilon^{\prime}$.
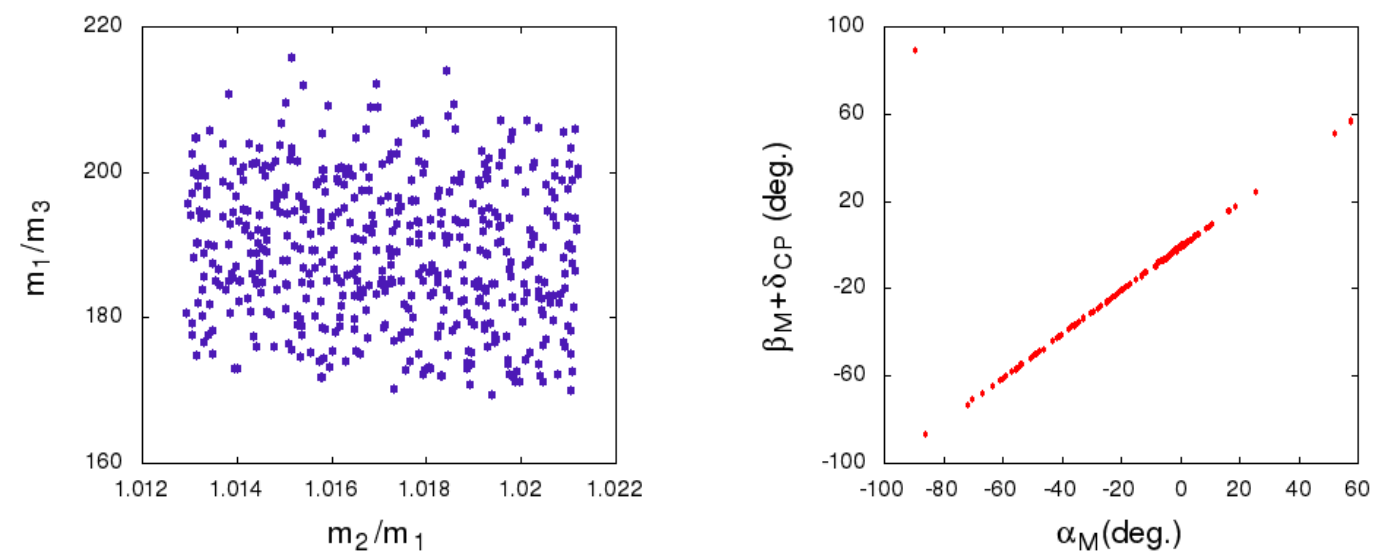

Figure 6. Plot of $\left(m_{1} / m_{3}\right)$ vs $\left(m_{2} / m_{1}\right)$ (left) and $\beta_{M}+\delta_{\mathrm{CP}}$ vs $\alpha_{M}$ (right) after breaking of the scaling ansatz in both the sectors of Category A for a representative value of $\epsilon=\epsilon^{\prime}=0.1$.

1. After precise determination of $\theta_{13}$ taking full account of reactor neutrino experimental data, it is shown that the hierarchy of the light neutrino masses can be probed through combined utilization of $\mathrm{NO} \nu \mathrm{A}$ and $\mathrm{T} 2 \mathrm{~K}$ [49] neutrino oscillation experimental results in near future. Thus the speculation of hierarchy in the present scheme will be clearly verified. Moreover, taking the difference of probabilities between $P\left(\nu_{\mu} \rightarrow \nu_{e}\right)$ and $P\left(\overline{\nu_{\mu}} \rightarrow \overline{\nu_{e}}\right)$ information on the value of $J_{\mathrm{CP}}$ can be obtained using neutrino and anti neutrino beams.

2. More precise estimation of the sum of the three light neutrino masses will be obtained utilizing a combined analysis with PLANCK data [50] and other cosmological and astrophysical experiments [51] such as, Baryon oscillation spectroscopic survey, The Dark energy survey, Large Synoptic Survey Telescope or the Euclid satellite data [52] etc. Such type of analysis will push $\Sigma_{i} m_{i} \sim 0.1 \mathrm{eV}$ (at the $4 \sigma$ level for inverted order- 
ing) and $\Sigma_{i} m_{i} \sim 0.05 \mathrm{eV}$ (at the $2 \sigma$ level for normal ordering). Thus the prediction of the value of $\Sigma_{i} m_{i}$ in the different categories discussed in the present work will also be tested in the near future. Furthermore, the NEXT-100 [53] will probe the value of $\left|m_{11}\right|$ up to $0.1 \mathrm{eV}$ which is a more precise estimation than the EXO-200 [54] experimental range $(0.14-0.38 \mathrm{eV})$.

\section{Summary and conclusion}

In this work we explore the phenomenology of neutrino mass matrix obtained due to inverse seesaw mechanism adhering i) Scaling ansatz, ii) Texture zeros within the framework of $\mathrm{SU}(2)_{L} \times \mathrm{U}(1)_{Y}$ model with three right handed neutrinos and three left chiral singlet fermions. Throughout our analysis we choose a basis in which the charged lepton mass matrix $\left(m_{E}\right)$ and the $M_{R S}$ matrix (appeared in inverse seesaw mechanism due to the coupling of $\nu_{R}$ and $S_{L}$ ) are diagonal. It is found that four is the maximum number of zeros that can be allowed in $m_{D}$ and $\mu$ matrices to obtain viable phenomenology. We classify different four zero textures in four different categories depending upon their generic form. Since scaling ansatz invariance always gives rise to $\theta_{13}=0$, we have to break such ansatz. We consider breaking in $m_{D}$ and also in $\mu$ matrices. We explore the parameter space and it is seen that one category (Category D) is ruled out phenomenologically. The hierarchy obtained in all the cases are inverted and it is interesting to note that all such categories give rise to tiny $C P$ violation measure $J_{\mathrm{CP}}$ due to small value of $\delta_{\mathrm{CP}}$. In conclusion, further observation of hierarchy of neutrino masses and $\mathrm{CP}$ violation in the leptonic sector in the forthcoming experiments will conclusively refute or admit all these categories obtained in the present scheme.

\section{Acknowledgments}

We thank Mainak Chakraborty for discussion and computational help.

Open Access. This article is distributed under the terms of the Creative Commons Attribution License (CC-BY 4.0), which permits any use, distribution and reproduction in any medium, provided the original author(s) and source are credited.

\section{References}

[1] R.N. Mohapatra and J.W.F. Valle, Neutrino Mass and Baryon Number Nonconservation in Superstring Models, Phys. Rev. D 34 (1986) 1642 [InSPIRE].

[2] J. Bernabeu, A. Santamaria, J. Vidal, A. Mendez and J.W.F. Valle, Lepton Flavor Nonconservation at High-Energies in a Superstring Inspired Standard Model, Phys. Lett. B 187 (1987) 303 [INSPIRE].

[3] R.N. Mohapatra, Mechanism for Understanding Small Neutrino Mass in Superstring Theories, Phys. Rev. Lett. 56 (1986) 561 [INSPIRE].

[4] J. Schechter and J.W.F. Valle, Neutrino Decay and Spontaneous Violation of Lepton Number, Phys. Rev. D 25 (1982) 774 [InSPIRE]. 
[5] A. Palcu, A straightforward realization of a quasi-inverse seesaw mechanism at TeV scale, arXiv: 1408.6518 [INSPIRE].

[6] J. Schechter and J.W.F. Valle, Neutrino Masses in $\mathrm{SU}(2) \times \mathrm{U}(1)$ Theories, Phys. Rev. D 22 (1980) 2227 [INSPIRE].

[7] S. Fraser, E. Ma and O. Popov, Scotogenic Inverse Seesaw Model of Neutrino Mass, Phys. Lett. B 737 (2014) 280 [arXiv: 1408.4785] [INSPIRE].

[8] H. Hettmansperger, M. Lindner and W. Rodejohann, Phenomenological Consequences of sub-leading Terms in See-Saw Formulas, JHEP 04 (2011) 123 [arXiv:1102.3432] [INSPIRE].

[9] B. Adhikary, A. Ghosal and P. Roy, Maximal zero textures of the inverse seesaw with broken $\mu \tau$ symmetry, Indian J. Phys. 88 (2014) 979 [arXiv:1311.6746] [INSPIRE].

[10] S.S.C. Law and K.L. McDonald, Generalized inverse seesaw mechanisms, Phys. Rev. D 87 (2013) 113003 [arXiv:1303.4887] [INSPIRE].

[11] P.S.B. Dev and A. Pilaftsis, Minimal Radiative Neutrino Mass Mechanism for Inverse Seesaw Models, Phys. Rev. D 86 (2012) 113001 [arXiv:1209.4051] [InSPIRE].

[12] M. Malinsky, Non-unitarity effects in the minimal inverse seesaw model, PoS (EPS-HEP 2009) 288 [arXiv: 0909.1953] [INSPIRE].

[13] M. Hirsch, S. Morisi and J.W.F. Valle, A4-based tri-bimaximal mixing within inverse and linear seesaw schemes, Phys. Lett. B 679 (2009) 454 [arXiv:0905.3056] [InSPIRE].

[14] D.V. Forero, M. Tortola and J.W.F. Valle, Neutrino oscillations refitted, Phys. Rev. D 90 (2014) 093006 [arXiv: 1405.7540] [INSPIRE].

[15] M.C. Gonzalez-Garcia, M. Maltoni, J. Salvado and T. Schwetz, Global fit to three neutrino mixing: critical look at present precision, JHEP 12 (2012) 123 [arXiv:1209.3023] [INSPIRE].

[16] D.V. Forero, M. Tortola and J.W.F. Valle, Global status of neutrino oscillation parameters after Neutrino-2012, Phys. Rev. D 86 (2012) 073012 [arXiv: 1205.4018] [INSPIRE].

[17] A.S. Joshipura and W. Rodejohann, Scaling in the Neutrino Mass Matrix, mu-tau Symmetry and the See-Saw Mechanism, Phys. Lett. B 678 (2009) 276 [arXiv:0905.2126] [INSPIRE].

[18] R.N. Mohapatra and W. Rodejohann, Scaling in the neutrino mass matrix, Phys. Lett. B 644 (2007) 59 [hep-ph/0608111] [INSPIRE].

[19] A. Blum, R.N. Mohapatra and W. Rodejohann, Inverted mass hierarchy from scaling in the neutrino mass matrix: low and high energy phenomenology, Phys. Rev. D 76 (2007) 053003 [arXiv: 0706.3801] [INSPIRE].

[20] M. Obara, The Possible Textures in the Seesaw Realization of the Strong Scaling Ansatz and the Implications for Thermal Leptogenesis, arXiv:0712.2628 [INSPIRE].

[21] A. Damanik, M. Satriawan, Muslim and P. Anggraita, Neutrino mass matrix from seesaw mechanism subjected to texture zero and invariant under a cyclic permutation, arXiv: 0705.3290 [INSPIRE].

[22] S. Goswami and A. Watanabe, Minimal Seesaw Textures with Two Heavy Neutrinos, Phys. Rev. D 79 (2009) 033004 [arXiv:0807.3438] [INSPIRE].

[23] W. Grimus and L. Lavoura, Softly broken lepton number $L_{e}-L_{\mu}-L_{\tau}$ with non-maximal solar neutrino mixing, J. Phys. G 31 (2005) 683 [hep-ph/0410279] [INSPIRE]. 
[24] M.S. Berger and S. Santana, Combined flavor symmetry violation and lepton number violation in neutrino physics, Phys. Rev. D 74 (2006) 113007 [hep-ph/0609176] [INSPIRE].

[25] S. Goswami, S. Khan and W. Rodejohann, Minimal Textures in Seesaw Mass Matrices and their low and high Energy Phenomenology, Phys. Lett. B 680 (2009) 255 [arXiv:0905.2739] [INSPIRE].

[26] S. Dev, R.R. Gautam and L. Singh, Charged Lepton Corrections to Scaling Neutrino Mixing, Phys. Rev. D 89 (2014) 013006 [arXiv:1309.4219] [inSPIRE].

[27] B. Adhikary, M. Chakraborty and A. Ghosal, Scaling ansatz, four zero Yukawa textures and large $\theta_{13}$, Phys. Rev. D 86 (2012) 013015 [arXiv: 1205.1355] [INSPIRE].

[28] P.H. Frampton, S.L. Glashow and D. Marfatia, Zeroes of the neutrino mass matrix, Phys. Lett. B 536 (2002) 79 [hep-ph/0201008] [INSPIRE].

[29] K. Whisnant, J. Liao and D. Marfatia, Constraints on texture zero and cofactor zero models for neutrino mass, AIP Conf. Proc. 1604 (2014) 273 [INSPIRE].

[30] P.O. Ludl and W. Grimus, A complete survey of texture zeros in the lepton mass matrices, JHEP 07 (2014) 090 [arXiv: 1406.3546] [INSPIRE].

[31] W. Grimus and P.O. Ludl, Correlations and textures in the neutrino mass matrix, PoS (EPS-HEP 2013) 075 [arXiv: 1309.7883] [INSPIRE].

[32] J. Liao, D. Marfatia and K. Whisnant, Texture and Cofactor Zeros of the Neutrino Mass Matrix, JHEP 09 (2014) 013 [arXiv:1311.2639] [INSPIRE].

[33] H. Fritzsch, Z.-z. Xing and S. Zhou, Two-zero Textures of the Majorana Neutrino Mass Matrix and Current Experimental Tests, JHEP 09 (2011) 083 [arXiv:1108.4534] [INSPIRE].

[34] A. Merle and W. Rodejohann, The elements of the neutrino mass matrix: allowed ranges and implications of texture zeros, Phys. Rev. D 73 (2006) 073012 [hep-ph/0603111] [InSPIRE].

[35] W. Wang, Neutrino mass textures with one vanishing minor and two equal cofactors, Eur. Phys. J. C 73 (2013) 2551 [arXiv:1306.3556] [inSPIRE].

[36] W. Wang, Parallel Texture Structures with Cofactor Zeros in Lepton Sector, Phys. Lett. B 733 (2014) 320 [Erratum ibid. B 738 (2014) 524] [arXiv: 1401.3949] [INSPIRE].

[37] L. Lavoura, Zeros of the inverted neutrino mass matrix, Phys. Lett. B 609 (2005) 317 [hep-ph/0411232] [INSPIRE].

[38] A. Kageyama, S. Kaneko, N. Shimoyama and M. Tanimoto, Seesaw realization of the texture zeros in the neutrino mass matrix, Phys. Lett. B 538 (2002) 96 [hep-ph/0204291] [INSPIRE].

[39] W. Wang, Parallel Lepton Mass Matrices with Texture and Cofactor Zeros, Phys. Rev. D 90 (2014) 033014 [arXiv:1402.6808] [INSPIRE].

[40] G.C. Branco, D. Emmanuel-Costa, M.N. Rebelo and P. Roy, Four Zero Neutrino Yukawa Textures in the Minimal Seesaw Framework, Phys. Rev. D 77 (2008) 053011 [arXiv: 0712.0774] [INSPIRE].

[41] S. Choubey, W. Rodejohann and P. Roy, Phenomenological consequences of four zero neutrino Yukawa textures, Nucl. Phys. B 808 (2009) 272 [arXiv:0807.4289] [InSPIRE].

[42] M. Chakraborty, H.Z. Devi and A. Ghosal, Scaling ansatz with texture zeros in linear seesaw, Phys. Lett. B 741 (2015) 210 [arXiv:1410.3276] [INSPIRE]. 
[43] B. Adhikary, A. Ghosal and P. Roy, $\mu \tau$ symmetry, tribimaximal mixing and four zero neutrino Yukawa textures, JHEP 10 (2009) 040 [arXiv:0908.2686] [INSPIRE].

[44] B. Adhikary, A. Ghosal and P. Roy, Baryon asymmetry from leptogenesis with four zero neutrino Yukawa textures, JCAP 01 (2011) 025 [arXiv:1009.2635] [INSPIRE].

[45] B. Adhikary, A. Ghosal and P. Roy, Neutrino masses, cosmological bound and four zero Yukawa textures, Mod. Phys. Lett. A 26 (2011) 2427 [arXiv:1103.0665] [InSPIRE].

[46] W. Grimus, A.S. Joshipura, L. Lavoura and M. Tanimoto, Symmetry realization of texture zeros, Eur. Phys. J. C 36 (2004) 227 [hep-ph/0405016] [INSPIRE].

[47] B. Adhikary, M. Chakraborty and A. Ghosal, Masses, mixing angles and phases of general Majorana neutrino mass matrix, JHEP 10 (2013) 043 [Erratum ibid. 09 (2014) 180] [arXiv: 1307.0988] [INSPIRE].

[48] Particle Data Group collaboration, J. Beringer et al., Review of Particle Physics (RPP), Phys. Rev. D 86 (2012) 010001 [inSPIRE].

[49] K. Ieki, Observation of $\nu_{\mu} \rightarrow \nu_{e}$ oscillation in the T2K experiment, Ph.D. Thesis, Kyoto University, Kyoto Japan (2014), T2K-THESIS-040.

[50] Planck collaboration, P.A.R. Ade et al., Planck 2013 results. XVI. Cosmological parameters, Astron. Astrophys. 571 (2014) A16 [arXiv:1303.5076] [InSPIRE].

[51] J. Lesgourgues and S. Pastor, Neutrino cosmology and Planck, New J. Phys. 16 (2014) 065002 [arXiv: 1404.1740] [INSPIRE].

[52] B.Audren et al., Neutrino masses and cosmological parameters from a Euclid-like survey: Markov Chain Monte Carlo forecasts including theoretical errors, JCAP 01 (2013) 026 [arXiv: 1210.2194] [INSPIRE].

[53] NEXT collaboration, D. Lorca, The Hunt for neutrinoless double beta decay with the NEXT experiment, arXiv:1411.0475 [INSPIRE].

[54] EXO collaboration, M. Auger et al., Search for Neutrinoless Double-Beta Decay in ${ }^{136}$ Xe with EXO-200, Phys. Rev. Lett. 109 (2012) 032505 [arXiv:1205.5608] [inSPIRE]. 